\title{
Erste Schuljahre im Schatten der Pandemie: Was haben Grundschulkinder erlebt und was kommt nun auf sie zu?
}

\author{
Catherine Gunzenhauser ${ }^{1,2}$, Henrik Saalbach ${ }^{2,3}$ \\ Albert-Ludwigs-Universität Freiburg \\ 2 Leipziger Forschungszentrum für frühkindliche Entwicklung, LFE \\ 3 Universität Leipzig
}

\begin{abstract}
Zusammenfassung: Das Bildungsangebot der Grundschulen ist seit Pandemiebeginn geprägt durch Einschränkungen des Präsenzunterrichts. Die einhergehende Qualitätsminderung kann den weiteren Bildungs- und Lebensweg von Grundschulkindern wesentlich beeinflussen. Im vorliegenden Beitrag fragen wir, welche Lernbedingungen Grundschulkinder während der Pandemie vorgefunden und wie diese auf ihre Kompetenzentwicklung gewirkt haben. Aktuelle Studien, die sich überwiegend auf Elternbefragungen stützen, zeichnen ein heterogenes Bild. Danach weist der Fernunterricht der Grundschulen ein geringes Potenzial an kognitiver Aktivierung einhergehend mit einem geringen Ausmaß an Rückmeldungen auf. Verlässliche Aussagen zur Wirkung des Fernunterrichtes sind nicht möglich, da während der Pandemie kaum Lernstandserhebungen durchgeführt wurden. Die vorliegenden Daten verweisen jedoch auf eine sehr hohe Varianz in den Leistungsentwicklungen. Wir diskutieren zudem Möglichkeiten, wie die Auswirkungen des eingeschränkten Unterrichts kompensiert werden können. So sollten nicht nur breitangelegte innovative Förderprogramme entwickelt, sondern auch der Übergang zu weiterführenden Schulen im Lichte der ungleich verteilten Einschränkungen angepasst werden. Die Forschung zu den lehr-lern-bezogenen Auswirkungen der Pandemie ist nicht nur für die Bewältigung der aktuellen Herausforderungen nützlich, sondern auch für den Erkenntnisgewinn zur Unterrichtsentwicklung und zur Verbesserung der individualisierten Förderung überhaupt.
\end{abstract}

Schlüsselbegriffe: Kompetenzentwicklung, Fernunterricht, COVID-19, Grundschule, Förderung

\section{First years of schooling during the pandemic: What did primary school students experience and what will they be facing now?}

\begin{abstract}
Summary: Teaching in primary school has been strongly affected by the pandemic. The resulting impairment of teaching quality can come along with long-term effects on children's education and well-being. In the present article, we thus ask what learning conditions primary school children have faced during the pandemic and how these may have affected the development of their academic competencies. We found that recent research, which is mostly based on parent survey, revealed very heterogeneous results. However, distance teaching during the pandemic seemed to be not very engaging and did not provide much individual feedback. Reliable conclusions concerning the consequence for learning are difficult to draw at this point as systematic assessments of academic performance were rare. Recent data, however, suggests a high variability in the development of academic skills. We further discuss possible means for compensation of the academic deficits due to the distance teaching. We not only suggest the development and implementation of innovative remedial teaching programs but also the adjustment of the transition to secondary schools to the differently affected learning conditions during the pandemic. Researching the consequences of the pandemic for learning and instruction not only helps coping with the current situation but may also promote the development of instructional methods and innovate means of individual learning support.
\end{abstract}

Keywords: Academic achievement, remote schooling, COVID-19, primary school, remedial teaching

Psychologie in Erziehung und Unterricht, 2021, 68, 280-286

DOI 10.2378/peu2021.art18d

(c) Ernst Reinhardt Verlag München Basel 
Wenn Grundschulkinder aufgrund der Pandemie weniger erfolgreich lernen als unter Regelbedingungen, kann dies ihren weiteren Bildungs- und Lebensweg sowohl aufgrund der Effekte mangelnden bereichsspezifischen Vorwissens als auch aufgrund der darauf basierenden Entscheidung für die weiterführende Schulart wesentlich beeinflussen (z. B. Ahmed, Tang, Waters \& DavisKean, 2019; Anger \& Plünnecke, 2021; Wößmann, 2020). Das Lernen unter Pandemie-Bedingungen ist im Grundschulalter eng mit der häuslichen Betreuung verzahnt, da Betreuungspersonen pädagogische Aufgaben übernehmen und die Kommunikation mit der Lehrkraft ermöglichen (cp. Langmeyer, Guglhör-Rudan, Naab, Urlen \& Winklhofer, 2020). Wir orientieren uns am Angebot-Nutzungs-Modell zur Erklärung des Lernerfolgs nach Helmke (2015). Unterschieden wird dabei zwischen dem Angebot (also dem Unterricht oder Äquivalenten während der Zeit der Schulschließungen), der Nutzung (also der aktiven Lernzeit oder selbstgesteuerten Lernaktivitäten) sowie der Wirkung, also dem Lernerfolg. Das Lernpotenzial der Kinder (also etwa bereichsspezifisches Vorwissen, Lernmotivation und selbstregulative Fähigkeiten) beeinflusst die Nutzung (cp. Helmke, 2015). Zudem werden u. a. strukturelle Merkmale der Familien als Einflüsse auf das Lernpotenzial der Kinder bzw. auf die Qualität des Angebots und die Intensität der Nutzung benannt (Helmke, 2015).

\section{Angebot und Nutzung im ersten Jahr der Pandemie}

Das schulische Bildungsangebot ist seit Beginn der Pandemie Anfang 2020 geprägt durch Einschränkungen des Präsenzunterrichts und Wechsel zwischen Organisationsformen (siehe Fickermann \& Edelstein, 2021). Bereits während des ersten Lockdowns 2020 besuchten Grundschulkinder durchschnittlich etwas länger die Schule als Schüler:innen weiterführender Schulen (Wößmann et al., 2020). Es ist jedoch davon auszugehen, dass es seit Frühjahr 2020 deutliche interindividuelle Unterschiede im Schulbesuch von Grundschulkindern gibt.

\section{Angebot in Phasen des Fernunterrichts}

Eine Umfrage unter etwa 1.800 Eltern von Grundschulkindern ergab, dass viele Grundschulen sich in der ersten Phase des Fernlernens auf die Kernfächer Deutsch und Mathematik konzentrierten; $\mathrm{zu}$ sachunterrichtsbezogenen Themen erhielten knapp $60 \%$ der Grundschulkinder Fernunterricht bzw. Arbeitsaufträge; zu den Bereichen Kunst, Musik und Sport jeweils weniger als $20 \%$ (Wildemann \& Hosenfeld, 2020). Zudem wurde an Grundschulen insgesamt deutlich seltener OnlineUnterricht angeboten als an weiterführenden Schulen (Wößmann et al., 2021). Allerdings verweisen die Erkenntnisse der empirischen Unterrichtsforschung darauf, dass sogenannte Sichtstrukturen des Unterrichts (also leicht beobachtbare Merkmale) für den Lernerfolg weniger ausschlaggebend sind als Tiefenstrukturen, also Methoden, die auf die zugrunde liegenden Lehr- und Lernprozesse zielen (cp. Decristan, Hess, Holzberger \& Praetorius, 2020). Für das Fernlernen würde dies bedeuten, dass das Medium der Vermittlung (z. B. digitale Lernplattform versus E-Mail-Kontakt) weniger wichtig für den Lernerfolg ist als etwa die Anregungsqualität des Fernunterrichts sowie die Qualität des Feedbacks durch die Lehrkraft (cp. Gunzenhauser, Enke, Johann, Karbach \& Saalbach, 2021; Voss \& Wittwer, 2020). Helm, Huber und Loisinger (2021) fassen über die Schularten hinweg zusammen, dass kognitiv anregende, herausfordernde Aufgaben wohl eher selten eingesetzt wurden. Für Grundschulkinder berichten Wildemann und Hosenfeld (2020), dass eine Mehrheit der befragten Eltern Aufgaben eher als wenig abwechslungsreich wahrnahmen. In Bezug auf das Vorhandensein hilfreichen Feedbacks durch die Lehrkräfte können Vermutungen daraus gezogen werden, wie häufig die Kinder überhaupt Kontakt zu Lehrkräften hatten und überhaupt Rückmeldung erhielten. Langmeyer et al. (2020) berichten etwa, dass laut Elternberichten ca. $8 \%$ der Grundschulkinder während des ersten Lockdowns gar keinen Kontakt und wei- 
tere $30 \%$ „selten“ Kontakt zu Lehrkräften hatten, während etwa $30 \%$ den Kontakt als „häufig“ oder „sehr häufig“ beschrieben. Angaben zur Häufigkeit von Rückmeldungen durch die Lehrkräfte unterscheiden sich zwischen Studien, wobei Lehrkräfte und Schüler:innen deutlich häufiger von Rückmeldung berichten als Eltern (Helm et al., 2021). In unseren Daten zu 67 Grundschulkindern gab etwa die Hälfte der Eltern während des ersten Lockdowns an, dass ihre Kinder von den Lehrkräften keine Rückmeldung zu den Deutsch- und Mathematikaufgaben erhielten (Gunzenhauser et al., 2021). Eltern, die von Rückmeldungen an ihre Kinder berichteten, schätzten diese in etwa $70 \%$ (Mathematik) bzw. $77 \%$ (Deutsch) der Fälle als „oft“ oder „immer“ hilfreich ein. Insgesamt deuten diese Ergebnisse darauf hin, dass die Qualität der durch die Lehrkräfte angeregten Lehr-Lernprozesse stark variierte.

\section{Nutzung in Phasen des Fernunterrichts}

Grundschulkinder in Deutschland verbrachten im Frühjahr 2020 durchschnittlich etwa 3,6 Stunden (Summe von Schulbesuch und Lernen für die Schule) und im Frühjahr 2021 etwa 4,1 Stunden pro Tag mit schulischen Aufgaben (Wößmann et al., 2020; Wößmann et al., 2021). Im Regelunterricht sehen die Stundentafeln - mit länderspezifischen Unterschieden etwa 25 Schulstunden (also knapp 19 Zeitstunden) pro Woche für Grundschulkinder vor (Ministerium für Kultus, Jugend und Sport Baden-Württemberg, 2021; Sächsische Staatskanzlei, 2020), wobei Hausaufgaben noch hinzukommen. Der zeitliche Umfang der Beschäftigung mit dem Lernstoff scheint sich also für Grundschulkinder in der Zeit des Fernunterrichts nicht extrem vom Umfang der Regelbeschulung unterschieden zu haben. Auch hier ist allerdings mit substanzieller Varianz etwa aufgrund von Unterschieden im Lernpotenzial der Kinder oder in Merkmalen des familiären Hintergrunds zu rechnen. In ihrem Literaturüberblick finden Helm et al. (2021) Zusammenhänge des soziodemografischen Hintergrundes unter anderem zu technischer Ausstattung und Unterstützung durch die Eltern, aber auch zu Motivation und Selbstständigkeit. Während des ersten Lockdowns 2020 haben Schüler:innen mit vergleichsweise guten selbstregulativen Fähigkeiten höhere Unabhängigkeit beim Lernen gezeigt (Blume, Schmidt, Kramer, Schmiedek \& Neubauer, 2021). Diese Faktoren beeinflussen die Nutzung des Lernangebots grundsätzlich bei Schüler:innen aller Altersgruppen. Die alterstypische Entwicklung der selbstregulativen Fähigkeiten (cp. Best \& Miller, 2010; Roebers, 2017) geht jedoch damit einher, dass viele Grundschulkinder noch Unterstützung bei der Regulation ihres Lernverhaltens benötigen. Kinder im Grundschulalter haben typischerweise noch Schwierigkeiten bei der Planung und Überwachung von Aufgaben (zusammenfassend siehe Roebers, 2017). Beispielsweise betrifft dies die Überwachung des eigenen Textverständnisses und die bedarfsorientierte Aufteilung der Lernzeit (z. B. de Bruin, Thiede, Camp \& Redford, 2011; Lockl \& Schneider, 2002).

\section{Wirkungen: Wie verändert die Pandemie-Situation die Entwicklung schulischer Fertigkeiten bei Grundschulkindern?}

Internationale Studien zeigen, dass längere Zeiträume ohne Beschulung (z. B. bedingt durch Streiks) oder auch längere Ferienzeiten sich negativ auf die Kompetenzentwicklung, aber auch langfristig auf Arbeitsmarktchancen auswirken können (Anger \& Plünnecke, 2021; Wößmann, 2020). Diese Ergebnisse sind auf die aktuelle Situation allerdings nur bedingt übertragbar, da die Grundschüler:innen ja nicht ohne Beschulung waren. Helm et al. (2021) fassen zusammen, dass unter befragten Schüler:innen, Eltern und Lehrkräften je nach Studie etwa ein Fünftel bis zwei Drittel der Befragten negative Auswirkungen der Schulschließungen auf den Lernerfolg erwarteten. Speziell für Grundschulkinder in Deutschland berichten Wößmann et al. (2021), dass etwa $52 \%$ nach Einschätzung ihrer Eltern während der Zeit des Fernunterrichts weniger 
lernten als in der Schule. Bisher liegen wenige empirische Studien vor, die die Leistungsentwicklung über den Zeitraum der Pandemie hinweg - speziell an Grundschulkindern - objektiv messen. Eine Studie aus Hamburg stellte unter Verwendung standardisierter Schulleistungstests aus den Jahren 2019 und 2020 fest, dass die Kinder des „Pandemie-Jahrgangs“ entgegen den Erwartungen keine signifikant schwächeren Leistungen zeigen (Depping, Lücken, Musekamp \& Thonke, 2021). Für Kinder, die im September 2020 in Baden-Württemberg in die fünfte Klasse (also in die erste Klasse der weiterführenden Schule) kamen, beobachteten Schult et al. (2021) in Daten einer standardisierten Lernstandserhebung einen leichten Rückgang der Lese- und Mathematikfähigkeiten im Vergleich zum Durchschnitt der drei vorigen Jahre. Bei Grundschulkindern aus der Schweiz zeigten Tomasik, Helbling und Moser (2020) einen Rückgang des Lernens in den Kernfächern während des Lockdowns im Vergleich zu einem Zeitraum vor dem Lockdown, wobei die Autoren hier beträchtliche individuelle Unterschiede im Lernzuwachs während des Lockdowns hervorheben. Diese Ergebnisse können nur einen ersten Einblick liefern, weisen aber bereits darauf hin, dass die genaue Diagnostik der Leistungsentwicklung von Grundschulkindern während der Pandemie unter Berücksichtigung individueller Einflussfaktoren ein wichtiges Feld für die empirische Bildungsforschung mit Implikationen für das Angebot an eventuellen kompensatorischen Bildungsangeboten nach Ende der Pandemie ist.

\section{Ausblick: Welche Aufträge ergeben sich für Forschung und Praxis nach Ende der Pandemie?}

Über das genaue Monitoring der Kompetenzentwicklung der Grundschulkinder hinaus ergeben sich aus unserer Sicht zwei Kernbereiche für nutzenorientierte Forschung und informierte Bildungspraxis nach Ende der Pandemie. Zum einen geht es um den Umgang mit möglichen Lernrückständen. Die Bundesregierung etwa hat hierfür eine Milliarde Euro in Aussicht gestellt, die zum Teil in Sommerprogramme sowie unterrichtsbegleitende Förderung investiert werden sollen (Bundesministerium für Bildung und Forschung und Bundesministerium für Familie, Senioren, Frauen und Jugend, 2021). Zum anderen muss speziell für Grundschulkinder der Übergang in die weiterführende Schule den Auswirkungen der Schulschließungen bzw. des eingeschränkten Schulbetriebs Rechnung tragen.

\section{Lernrückstände aufholen}

Unter dem Blickwinkel der Nutzung stellt sich die Frage, für wen die Teilnahme an Fördermaßnahmen sinnvoll wäre. Erste Daten lassen auf große Varianz im Ausmaß der Lernrückstände schließen (Tomasik et al., 2020). Für die pädagogische Praxis sind Leitlinien sinnvoll, für welche Kinder eine Teilnahme an Förderprogrammen empfohlen werden soll. Eine naheliegende Zielgruppe für Fördermaßnahmen sind die Grundschulkinder, deren Leistung nicht ihrer Klassenstufe entspricht. Aufgrund der quantitativen Ergebnisse (Schult, Mahler, Fauth \& Lindner, 2021) ist aber zu erwarten, dass es viele Kinder gibt, deren Leistung nach der Pandemie zwar den Standards ihrer Klassenstufe entspricht, sich aber weniger günstig entwickelt hat, als es unter Bedingungen der Regelbeschulung der Fall gewesen wäre. Für ein einzelnes Kind ist dies selbstverständlich nie mit Sicherheit nachzuweisen. Sinnvoll scheint es aber, dass der Zugang zu Fördermaßnahmen möglichst breit ermöglicht wird, damit alle Kinder in der Entfaltung ihres Potenzials unterstützt werden. Durch formative Leistungsmessungen über die kommenden Schuljahre hinweg kann bewertet werden, wie gut die Kompensationsangebote wirken, welche Kinder profitieren und welche die Förderung möglicherweise nicht länger benötigen. Ein Verständnis des „Aufholprogramms" als an alle Kinder gerichtete Maßnahme hätte außerdem den Vorteil, dass er einer Stigmatisierung der teilnehmenden Kinder entgegenwirkt. Wenn Kinder ein schwaches aka- 
demisches Fähigkeitsselbstkonzept entwickeln (sich also selbst als wenig leistungsfähig wahrnehmen), kann dies ihren zukünftigen Lernerfolg mindern (Guay, Marsh \& Boivin, 2003). Die Entwicklung des akademischen Fähigkeitsselbstkonzepts wird im Grundschulalter durch soziale Vergleiche beeinflusst (cp. Zeinz \& Köller, 2006) und hängt außerdem von Attributionen der eigenen Erfolge oder Misserfolge ab (cp. Wiegfield \& Eccles, 2000). Im Umgang mit Kindern sollte daher sichergestellt werden, dass diese ihre Teilnahme an Förderprogrammen nicht als Folge persönlichen schulischen Versagens wahrnehmen, sondern dass ihnen durch Lehrkräfte und Eltern deutlich gemacht wird, dass es die Schule ist, die hier etwas nachholt: die Lernangebote zu machen, die sie ihnen während der Pandemie nicht machen konnte.

Um eine substanzielle nachhaltige und breite Wirksamkeit der Förderprogramme zu ermöglichen, ist es notwendig, diese langfristig anzulegen. Das heißt, sie sollten sich nicht auf Ferienzeiten konzentrieren, sondern für mindestens zwei Jahre in den Schulbetrieb etwa im Rahmen von Ganztagsangeboten integriert werden. Aus unserer Sicht ist es zudem sehr wichtig, dass das Förderprogramm nicht losgelöst vom regulären Fachunterricht durchgeführt wird. Das heißt, dass die jeweilige Fachlehrkraft die koordinative Rolle im Hinblick auf die in der Förderung zu erarbeitenden Lernziele und Aufgaben übernimmt. Das heißt, durch eine regelmäßig durchgeführte formative Leistungsbewertung wird der Lern- bzw. Übungsbedarf ermittelt und an die Verantwortlichen des entsprechenden Förderangebots übermittelt. Als Medium zur Kommunikation nicht nur zwischen Klassen- bzw. Fachlehrkraft und der förderverantwortlichen Person, sondern auch zwischen Lehrkraft und den Eltern könnten die im letzten Jahr vielfach eingesetzten digitalen Klassenzimmer bzw. digitale Lernumgebungen genutzt werden, auf denen die Materialien und Aufgaben zur Förderung einsehbar sind, sodass diese ggf. auch von Eltern genutzt werden können. Personenbezogene Daten zum Lernstand wären entsprechend da- tenschutzrechtlich gesichert. Um den personellen Bedarf der Förderprogramme zu sichern, sind innovative und kreative Lösungen notwendig, die allerdings die für eine effektive Förderung notwendige Professionalität berücksichtigen müssen. Hierfür böte es sich an, gezielt Lehramtsstudierende anzusprechen und Möglichkeiten der Anrechenbarkeit der förderpädagogischen Arbeit auf die Studienleistungen (z. B. Praktika) in Aussicht zu stellen. Auch die Gewinnung von pensionierten Lehrkräften sollte erwogen werden.

\section{Der Übergang in die weiterführende Schule}

Die Entscheidung für eine weiterführende Schulart am Ende der Grundschulzeit ist im deutschen Bildungssystem eine wichtige Weichenstellung für den weiteren Bildungsweg. Die derzeitigen Kompetenzen von Grundschulkindern am Ende der Grundschulzeit wurden jedoch nicht unter Regelbeschulung erworben und können daher nicht im gewohnten Maß als valide Prädiktoren der Leistungsentwicklung in der weiterführenden Schule betrachtet werden. Besonders für die Schüler:innen, die die empfohlene Leistung für eine bestimmte weiterführende Schulart im letzten Grundschuljahr knapp nicht erreichen, wird die Entscheidung also erschwert. Insofern ist es derzeit - wie in den Regelungen zur Erstellung der Grundschulempfehlung angelegt (z. B. Ministerium für Kultus, Jugend und Sport Baden-Württemberg, 2019) - besonders wichtig, dass die pädagogischen Fachkräfte in ihrer Empfehlung die Entwicklung des Kindes über die gesamte Grundschulzeit hinweg sowie ihre Erfahrungen mit dem Lern- und Arbeitsverhalten des Kindes einbeziehen. Wenn für ein Kind eine klare Empfehlung schwerfällt, ist es außerdem sinnvoll, das Kind und die Eltern für die bestehenden Übergangsmöglichkeiten zwischen den weiterführenden Schularten zu sensibilisieren und den Zugang hierzu durch entsprechende Informations- und Beratungsangebote in den ersten Jahren der weiterführenden Schule zu erleichtern. 


\section{Abschließende Bemerkung}

Im Fokus steht zunächst die Kompensation von Lernrückständen durch die Pandemie und die Vermeidung ungünstiger Langzeitfolgen der Schulschließungen. Dennoch ist es angesichts der breiten Varianz in der Lernentwicklung während der Schulschließungen (z. B. Tomasik et al., 2020) nicht unwahrscheinlich, dass manche Grundschulkinder von der Fernlehre sogar profitiert haben. Eine Frage für die Bildungsforschung ist es daher auch, welche Faktoren aufseiten des Angebots und aufseiten der Nutzung eine solche Entwicklung befördert haben könnten. Perspektivisch könnten diese Erkenntnisse zur Unterrichtsentwicklung und zur Verbesserung der individualisierten Förderung genutzt werden.

\section{Literatur}

Ahmed, S. F., Tang, S., Waters, N. E. \& Davis-Kean, P. (2019) Executive function and academic achievement: Longitudinal relations from early childhood to adolescence. Journal of Educational Psychology, 111, 446-458.

Anger, C. \& Plünnecke, A. (2021). Bildungsgerechtigkeit: Herausforderung für das deutsche Bildungssystem (No. 140). IW-Analysen.

Best, J. R. \& Miller, P. H. (2010). A developmental perspective on executive function. Child Development, 81 $1641-1660$.

Blume, F., Schmidt, A., Kramer, A. C., Schmiedek, F. \& Neubauer, A. B. (2021). Homeschooling during the SARS CoV-2 pandemic: The role of students' trait self-regulation and task attributes of daily learning tasks for students' daily self-regulation. Zeitschrift für Erziehungswissenschaft, 24, 367-391.

Bundesministerium für Bildung und Forschung und Bundesministerium für Familie, Senioren, Frauen und Jugend (2021). Aktionsprogramm Aufholen nach Corona für Kinder und Jugendliche für die Jahre 2021 und 2022 - 2 Mrd. Euro. Zugriff am 14.6.2021 unter https://www.bmbf.de/files/BMFSFJ_Corona_Aufholpaket_Paper_06_sa\%20(1).pdf

de Bruin, A. B., Thiede, K. W., Camp, G. \& Redford, J. (2011). Generating keywords improves metacomprehension and self-regulation in elementary and middle school children. Journal of Experimental Child Psychology, 109, 294-310.

Decristan, J., Hess, M., Holzberger, D. \& Praetorius, A.-K. (2020). Oberflächen- und Tiefenmerkmale. Eine Reflexion zweier prominenter Begriffe der Unterrichtsforschung. Zeitschrift für Pädagogik, 66, 102-116.

Depping, D., Lücken, M., Musekamp, F. \& Thonke, F. (2021). Kompetenzstände Hamburger Schüler*innen vor und während der Corona-Pandemie. In D. Fickermann \& B. Edelstein (Hrsg.), Schule während der Corona-Pandemie. Neue Ergebnisse und Überblick über ein dynamisches Forschungsfeld Die Deutsche Schule, Beiheft 17, 51-79. Münster: Waxmann Verlag $\mathrm{GmbH}$.

Fickermann, D. \& Edelstein, B. (2021). Editorial des Beihefts Schule während der Corona-Pandemie: Neue Ergebnisse und Überblick über ein dynamisches Forschungsfeld. Die Deutsche Schule, Beiheft 17, 7-30.

Guay, F., Marsh, H. W. \& Boivin, M. (2003). Academic self-concept and academic achievement: Developmental perspectives on their causal ordering. Journal of Educational Psychology, 95, 124-136.

Gunzenhauser, C., Enke, S., Johann, V., Karbach, J. \& Saalbach, H. (2021). Parent and teacher support of elementary students' remote learning during the COVID-19 Pandemic. Manuscript submitted for publication.

Helm, C., Huber, S. \& Loisinger, T. (2021). Was wissen wir über schulische Lehr-Lern-Prozesse im Distanzunterricht während der Corona-Pandemie? - Evidenz aus Deutschland, Österreich und der Schweiz. Zeitschrift für Erziehungswissenschaft, 24, 237-311.

Helmke, A. (2015). Unterrichtsqualität und Lehrerprofessionalität. Diagnose, Evaluation und Verbesserung des Unterrichts. 6. überarbeitete Auflage. Seelze: Klett-Kallmeyer.

Langmeyer, A., Guglhör-Rudan, A., Naab, T., Urlen, M. \& Winklhofer, U. (2020). Kindsein in Zeiten von Corona. Erste Ergebnisse zum veränderten Alltag und zum Wohlbefinden von Kindern. Zugriff am 9.6.2021 unter: https://www.dji.de/fileadmin/user_upload/dasdji/the men/Familie/DJI_Kindsein_Corona_Erste_Ergebnisse. $\mathrm{pdf}$

Lockl, K. \& Schneider, W. (2002). Zur Entwicklung des selbstregulierten Lernens im Grundschulalter: Zusammenhänge zwischen Aufgabenschwierigkeit und Lernzeiteinteilung. Psychologie in Erziehung und Unterricht, $49,3-16$.

Ministerium für Kultus, Jugend und Sport Baden-Württemberg (2019). Grundschule: Von der Grundschule in die weiterführende Schule. Zugriff am 14.6.2021 unter https://km-bw.de/site/pbs-bw-km-root/get/docu ments_E-695614930/KULTUS.Dachmandant/KUL TUS/KM-Homepage/Publikationen\%202019/2019 $\% 20$ Grundschule \%20SCREEN.pdf

Ministerium für Kultus, Jugend und Sport Baden-Württemberg (2021). Kontingentstundentafel Grundschule. Zugriff am 20.5.2021 unter https://km-bw.de/,Lde/Start seite/Schule/Kontingentstundentafel+Grundschule

Roebers, C. M. (2017). Executive function and metacognition: Towards a unifying framework of cognitive selfregulation. Developmental Review, 45, 31-51.

Sächsische Staatskanzlei (2020). Stundentafeln Grundschulen. Zugriff am 14.6.2021 unter https://www.revosax. sachsen.de/vorschrift/17744-VwV-Stundentafeln\#xanl

Schult, J., Mahler, J., Fauth, B. \& Lindner, M. A. (2021). Did Students Learn Less During the COVID-19 Pandemic? Reading and Mathematics Competencies Before and After the First Pandemic Wave. Preprint. https://doi.org/10. 31234/osf.io/pqtgf

Tomasik, M. J., Helbling, L. A. \& Moser, U. (2020). Educational gains of in-person vs. distance learning in primary and secondary schools: A natural experiment during the COVID-19 pandemic school closures in Switzerland. International Journal of Psychology. Advance online publication.

Voss, T. \& Wittwer, J. (2020). Unterricht in Zeiten von Corona: Ein Blick auf die Herausforderungen aus der Sicht 
von Unterrichts- und Instruktionsforschung. Unterrichtswissenschaft, 48, 601-627.

Wigfield, A. \& Eccles, J. (2000). Expectancy-value theory of achievement motivation. Contemporary Eductional Psychology, 25, 68-81.

Wildemann, A. \& Hosenfeld, I. (2020). Bundesweite Elternbefragung zu Homeschooling während der Covid 19-Pandemie. Erkenntnisse zur Umsetzung des Homeschoolings in Deutschland. Zugriff am 14.6.2021 unter http://www.zepf.eu/wp-content/uploads/2020/06/ Bericht_HOMEschooling2020.pdf

Wößmann, L. (2020). Folgekosten ausbleibenden Lernens: Was wir über die Corona-bedingten SchulschlieBungen aus der Forschung lernen können. ifo Schnelldienst, 73(06), 38-44.

Wößmann, L., Freundl, V., Grewenig, E., Lergetporer, P., Werner, K. \& Zierow, L. (2020). Bildung in der Coronakrise: Wie haben die Schulkinder die Zeit der Schulschließungen verbracht, und welche Bildungsmaßnahmen befürworten die Deutschen? ifo Schnelldienst 73(09), 25-39.

Wößmann, L., Freundl, V., Grewenig, E., Lergetporer, P., Werner, K. \& Zierow, L. (2021). Bildung erneut im Lockdown: Wie verbrachten Schulkinder die Schulschließungen Anfang 2021? ifo Schnelldienst, $74(05)$, $36-52$.

Zeinz, H. \& Köller, O. (2006). Noten, soziale Vergleiche und Selbstkonzepte in der Grundschule. In Risikofaktoren kindlicher Entwicklung (pp. 177-190). VS Verlag für Sozialwissenschaften.

\section{JProf. Dr. Catherine Gunzenhauser \\ Universität Freiburg \\ Institut für Erziehungswissenschaft \\ Rempartstr. 11 \\ 79098 Freiburg \\ Leipziger Forschungszentrum \\ für frühkindliche Entwicklung (LFE) \\ E-Mail: catherine.gunzenhauser@ezw.uni-freiburg.de}

\section{Prof. Dr. Henrik Saalbach}

Universität Leipzig

Erziehungswissenschaftliche Fakultät

Marschnerstr. 31

04109 Leipzig

Leipziger Forschungszentrum

für frühkindliche Entwicklung (LFE)

E-Mail: henrik.saalbach@uni-leipzig.de 